\title{
Direct cell-to-cell interactions between osteocytes and multiple myeloma (MM) cells up-regulate Sost and down-regulate OPG expression in osteocytes: evidence for osteocytic contributions to MM- induced bone disease
}

\author{
Delgado-Calle $\mathrm{J}^{1,2,4}$, Bellido $\mathrm{T}^{2,3,4}$ and Roodman $\mathrm{GD}^{1,4}$ \\ ${ }^{1}$ Department of Hematology Oncology, ${ }^{2}$ Department of Anatomy and Cell Biology, ${ }^{3}$ Department of \\ Medicine, Division of Endocrinology, Indiana University School of Medicine. ${ }^{4}$ Roudebush Veterans \\ Administration Medical Center, Indianapolis, IN. \\ Indiana University-Purdue University Indianapolis
}

Osteocytes are the most abundant bone cells, comprising more than $95 \%$ of the cells in bone. They are embedded into the bone matrix, but extensively communicate among themselves and with cells on the bone surface and the bone marrow through the osteocytic lacunar-canalicular network. Osteocytes secrete sclerostin, the product of the Sost gene, an antagonist of Wnt signaling that potently inhibits bone formation. Osteocytes are also a major source of pro- and anti-osteoclastogenic cytokines that regulate osteoclastogenesis and bone resorption, including RANKL and osteoprotegerin (OPG). Recent evidence suggests that the bone remodeling compartment is disrupted in multiple myeloma (MM) allowing close contact of MM cells with bone cells including osteocytes. However, the consequences of these interactions and the contribution of osteocytes to MM bone disease are unclear. Therefore, we determined if interactions between MM cells and osteocytes regulate osteocytic gene expression. We found that co-culture of murine MLO-A5 osteocytic cells with human JJN3 MM cells up-regulated murine Sost mRNA expression 2-3 fold as early as $4 \mathrm{~h}$, which remained elevated up to $24 \mathrm{~h}$. Consistent with Sost up-regulation induced by MM cells, the expression of OPG, a Wnt target gene, was decreased by $30-50 \%$ in MLO-A5 cells, resulting in an increased RANKL/OPG at 4h. Culture of JJN3 cells in the top and MLO-A5 cells in the bottom of Boyden chambers abolished both up-regulation of Sost and down-regulation of OPG mRNA expression in osteocytic cells, demonstrating the requirement of direct contact between MM cells and osteocytic cells. Human Sost and OPG mRNA transcripts were not detected in any of these experiments, demonstrating lack of contribution of MM JJN3 cells. These findings demonstrate that direct interactions between osteocytes and MM cells up-regulate the expression of the bone formation inhibitor Sost in osteocytes, which in turn decreases Wnt signaling, reduces osteocytic OPG expression increasing the RANKL/OPG ratio. We propose that increased Sost/Sclerostin expression contributes to the exacerbated bone resorption and the decreased bone formation that characterizes MM induced bone disease. 